\title{
Defining malaria burden from morbidity and mortality records, self treatment practices and serological data in Magugu, Babati District, northern Tanzania
}

\author{
CHARLES MWANZIVA ${ }^{1 *}$, ALPHAXARD MANJURANO2, ERASTO MBUGI ${ }^{3}$, CLEMENT MWEYA4, \\ HUMPHREY MKALI ${ }^{5}$, MAGGIE P. KIVUYO ${ }^{6}$, ALEX SANGA ${ }^{7}$, ARNOLD NDARO ${ }^{1}$, WILLIAM CHAMBO, \\ ABAS MKWIZU ${ }^{9}$, JOVIN KITAU ${ }^{1}$, REGINALD KAVISHE ${ }^{1} 1$, WIL DOLMANS ${ }^{10}$, JAFFU CHILONGOLA ${ }^{1}$ \\ and FRANKLIN W. MOSHA ${ }^{1}$ \\ ${ }^{1}$ Kilimanjaro Clinical Research Institute, P. O. Box 2236, Moshi, Tanzania \\ 2Joint Malaria Programme, Moshi, Tanzania \\ ${ }^{3}$ Muhimbili University of Health and Allied Sciences, Dar es Salaam, Tanzania \\ ${ }^{4}$ Tukuyu Medical Research Centre, Tukuyu, Tanzania \\ ${ }^{5}$ Tabora Medical Research Centre, Tabora, Tanzania \\ ${ }^{6}$ Ngongongare Medical Research Station, Usa River, Tanzania \\ ${ }^{7}$ St. John University of Tanzania, Dodoma, Tanzania \\ ${ }^{8}$ Amani Medical Research Centre, Muheza, Tanzania \\ ${ }^{9}$ Magugu Health Centre, Babati, Manyara, Tanzania \\ ${ }_{10}$ Radboud University Nijmegen Medical Centre, Nijmegen, The Netherlands
}

Abstract: Malaria morbidity and mortality data from clinical records provide essential information towards defining disease burden in the area and for planning control strategies, but should be augmented with data on transmission intensity and serological data as measures for exposure to malaria. The objective of this study was to estimate the malaria burden based on serological data and prevalence of malaria, and compare it with existing self-treatment practices in Magugu in Babati District of northern Tanzania. Prospectively, 470 individuals were selected for the study. Both microscopy and Rapid Diagnostic Test (RDT) were used for malaria diagnosis. Seroprevalence of antibodies to merozoite surface proteins (MSP119) and apical membrane antigen (AMA-1) was performed and the entomological inoculation rate (EIR) was estimated. To complement this information, retrospective data on treatment history, prescriptions by physicians and use of bed nets were collected. Malaria prevalence in the area was $6.8 \%(32 / 470)$. Of 130 individuals treated with artemisinin combination therapy (ACT), $22.3 \%(29 / 130)$ were slide confirmed while $75.3 \%$ (98/130) of them were blood smear negative. Three of the slides confirmed individuals were not treated with ACT. Fever was reported in $38.2 \%$ of individuals, of whom $48.8 \%(88 / 180)$ were given ACT. Forty-two (32.3\%) of those who received ACT had no history of fever. About half $(51.1 \%)$ of those treated with ACT were children $<10$ years old. Immunoglobulin against MSP-1 19 was positive in $16.9 \%$ (74/437) while against AMA-1 was positive in $29.8 \%$ (130/436). Transmission intensity was estimated at $<0.2$ infectious bites per person per year. The RDT was highly specific $(96.3 \%)$ but with low sensitivity $(15.6 \%)$. In conclusion, Magugu is a low endemic area. There is substantial over diagnosis, over treatment and self treatment in the community. The burden of malaria based on medical records is over estimated as was mostly presumptive. The low sensitivity of RDT reflects the low number of immune individuals as well as the low parasite density.

Keywords: malaria, morbidity, mortality, diagnosis, treatment, Tanzania

\footnotetext{
* Correspondence: Dr. Charles Mwanziva; E-mail: cmwanziva@yahoo.com
} 


\section{Introduction}

Every year an estimated 300-600 million cases of malaria occur globally, resulting in 1 million deaths. Approximately $90 \%$ of malaria cases and more than $85 \%$ of deaths occur in Sub-Saharan Africa, mostly among children under the age of five (Crawley et al., 2010). Over much of the SubSaharan Africa, malaria transmission intensity varies from one area to another. Repeated exposure to malaria parasites and transmission intensity govern the development of acquired immunity, which controls the erythrocytic cycle of malaria, thereby reducing clinical symptoms and life-threatening complications. Merozoite antibodies play an important role in protecting erythrocyte invasion and opsonising merozoites for phagocytoses (Murhandarwati et al., 2008). Where malaria is endemic, individuals get continuous exposure, thus clinical manifestations in adults are low grade though these individuals may have parasitaemia (Andersen et al,. 1997; Bruce-Chwatt, 1952; Warsame et al., 1997). In non-endemic areas progressive lowering of acquired immunity is experienced (Luxemburger et al., 1997) but even then serological markers are maintained. These markers (Craig et al., 1999) are not influenced by the seasonal fluctuation in malaria incidences and are a potential tool for defining the malaria transmission intensity of the area (Bousema et al., 2010).

A number of programmes have been implemented to fight malaria in SSA, in three different or combined approaches: use of antimalarial drugs, vector control (elimination of mosquito breeding sites, indoor residual spraying and use of insecticide treated nets - ITN) and mass drug administration (MDA). These interventions resulted in a decline of malaria in many areas in Sub-Saharan Africa including Tanzania (Bhattarai et al., 2007; Barnes et al., 2009).

Contradicting reports still indicate high malaria incidences in Sub-Saharan Africa (Besnard et al., 2006; WHO, 2009), especially in rural areas where adequate diagnosis and prompt treatment continue to present major difficulties. Presumptive treatment in a malaria declining era (Gosling et al., 2008), has limited application. Over-diagnosis (Elgayoum et al., 2009) and overtreatment of malaria (Chandler et al., 2008) may lead to missing other causes of febrile illnesses. This may result in overuse of antimalarial drugs (Mwanziva et al., 2008) and increases the chance of developing drug resistance (Maude et al., 2009; White, 2010). Furthermore, self-treatment (Nsimba \& Rimoy, 2005; Hodel et al., 2009) and mistreatment of febrile illnesses in health facilities results into increasing hospitalization and deaths due to diseases other than malaria. The objective of this study was to estimate the malaria burden based on serological data and prevalence of malaria, and compare it with existing self-treatment practices in the community and in medical practice in Magugu, a malaria endemic area of northern Tanzania.

\section{Materials and Methods}

\section{Study site}

Magugu ward (S3099' S4001'; E35070' E35077') in Babati District is located in northern Tanzania along the rift valley, at approximately $900 \mathrm{~m}$ to $1200 \mathrm{~m}$. The ward has a total population of 26,131 people residing in seven villages. There is one government owned health centre which serves all the seven villages. Babati District is one of the eight sentinel sites in Tanzania for monitoring antimalarial drug efficacy. Livestock and crop agriculture (mainly rice, maize, sugar cane and 
vegetables) are the main occupations of the population. Average annual rainfall is about $650 \mathrm{~mm}$. There are two rainy seasons, short rainy season from October to December and long rainy season from mid March to May, followed by a cool and dry season from June to mid August, and a hot dry period from mid August to October.

\section{Data collection}

Retrospectively, medical records covering 2005-2007 from Babati Hospital and Magugu Health Centre were reviewed. The record comprised overall record of malaria morbidity of all patients attending the two facilities. Prospectively, individuals attending the facilities from June to August 2009 were asked to participate in the study regardless of their main complaint. A structured questionnaire was administered to record participant's information including age, gender, date of attendance and use of mosquito bed nets. Information was also collected on type of medication (antimalarials and other medicines) used in the previous two weeks. Body temperature, request for blood slide, rapid diagnostic test (RDT) and their results were recorded. Clinical diagnosis and treatment given before leaving the health facility was documented.

Three drops of blood were collected from a single finger prick and examined for malaria infection. The first drop was used for microscopic parasite detection; both for a thin and thick blood smear, and stained with 10\% Giemsa. Parasite density was estimated by counting the number of asexual parasites per 200 white blood cells (WBC) and the number of sexual forms per 500 WBC. A slide was judged negative if no parasites were seen after inspection of 100 high power fields. For quality control, a second microscopist, who was unaware of the results from the first reading, re-read all the slides. The second drop of blood was used to determine the presence of malaria parasites by RDT (Paracheck-Pf®, Orchid Biomedical Systems).

A third drop of blood was collected on Whatman's 3mm filter paper (Whitman, Maidstone, UK) for serological tests. Serum was eluted from filter papers as described by Corran et al. (2008). Immunoglobulin G antibodies against blood stage antigens were determined by indirect enzyme-linked immunosorbent assay (indirect ELISA), as previously described (Drakeley et al., 2005) using recombinant MSP-1 19 (Wellcome Genotype), produced as previously described by Franks et al. (2003) or recombinant AMA-1 (3D7). Briefly, flat bottom 96-well microtitre plates (Nunc, Roskilde, Denmark) were coated overnight with $50 \mu \mathrm{l}$ of $0.5 \mathrm{mg} / \mathrm{ml}$ dilution of the specific antigen. After washing with PBS-0.05\% Tween [(PBS-T), $200 \mu \mathrm{L}$ of blocking buffer (1\% skimmed milk [Marvel, Spalding, Lincs, UK] in PBS-T) was added for 3 hours at room temperature. After washing, $50 \mu \mathrm{L}$ sera with a dilution of 1:1000 for MSP-119, and 1:2000 for AMA-1, were added to the plate in duplicate and incubated for 3 hours at room temperature. Antigen-specific antibodies were detected using rabbit anti-human IgG (DAKO A/S Denmark) in a 1:5000 dilution and incubated for 20 minutes at room temperature. Plates were developed with o-phenyline-diamine (OPD) [Sigma]- $\mathrm{H}_{2} \mathrm{O} 2$ and the reaction was stopped with $50 \mu 1 \mathrm{H}_{2} \mathrm{SO} 4$. Plates were read at a wave length of $490 \mathrm{~nm}$ and the Optical Density (OD) values recorded.

\section{Data analysis}

Statistical analysis was carried out using SPSS 16.0 for windows (SPSS Inc, Chicago, USA). Proportions for categorical data were reported, and the Pearson Chi-squared test was used to assess the relationship between groups. Fisher 's exact test was used in cases of less than 5 counts. 
Standard deviation (SD) was used to describe the spread around the mean. A p-value $<0.05$ was considered statistically significant. To generate an Optical Density (OD) cut-off value above which samples were deemed antibody positive, the distribution of OD values was fitted as the sum of two Gaussian distributions (assuming a narrow distribution of seronegatives and a broader distribution of seropositives) using maximum likelihood methods. The mean OD of the Gaussian distribution corresponding with the seronegative population plus 3SD was used as the cut-off point (Corran et al., 2008). The OD values were expressed as the percentage of the positive control. The seroconversion rate (SCR or $\lambda$ ) was estimated by fitting a simple reversible catalytic model to the measured seroprevalence, stratified into yearly age groups, using maximum likelihood methods. For these models only individuals aged one year and above were included to remove the effects of the maternally derived antibodies in infants. The $\lambda$ values were converted to EIR equivalents using a log regression based on previously collected EIR values from areas of different transmission intensity in Tanzania (Drakeley et al., 2005; Corran et al., 2008).

\section{Ethical considerations}

Informed consent was obtained from all participants participating in prospective data collection (questionnaire and blood sampling). Only consenting individuals were included. The treatment practice by the medical personnel was not interfered with by the research activity. All results, including those of the RDT were accessible by the medical personnel as our study was observational.

\section{Results}

Data collected retrospectively at the sentinel site at Babati Hospital for the years 2005, 2006 and 2007 showed a small increase in malaria prevalence from 2005 (33.9\%) to the years 2006-2007 (41.5\% and 40.5\%, respectively). Records from Magugu HC (Figure 1) show a similar trend with increase of the burden of malaria during 2006 and 2007 in the area. Ten deaths were reported in 2006 at the Magugu health facility, 3 were children under the age of five. In 2007, 13 deaths were reported, of which 3 were children under the age of five. On both occasions cause of death was associated with malaria.

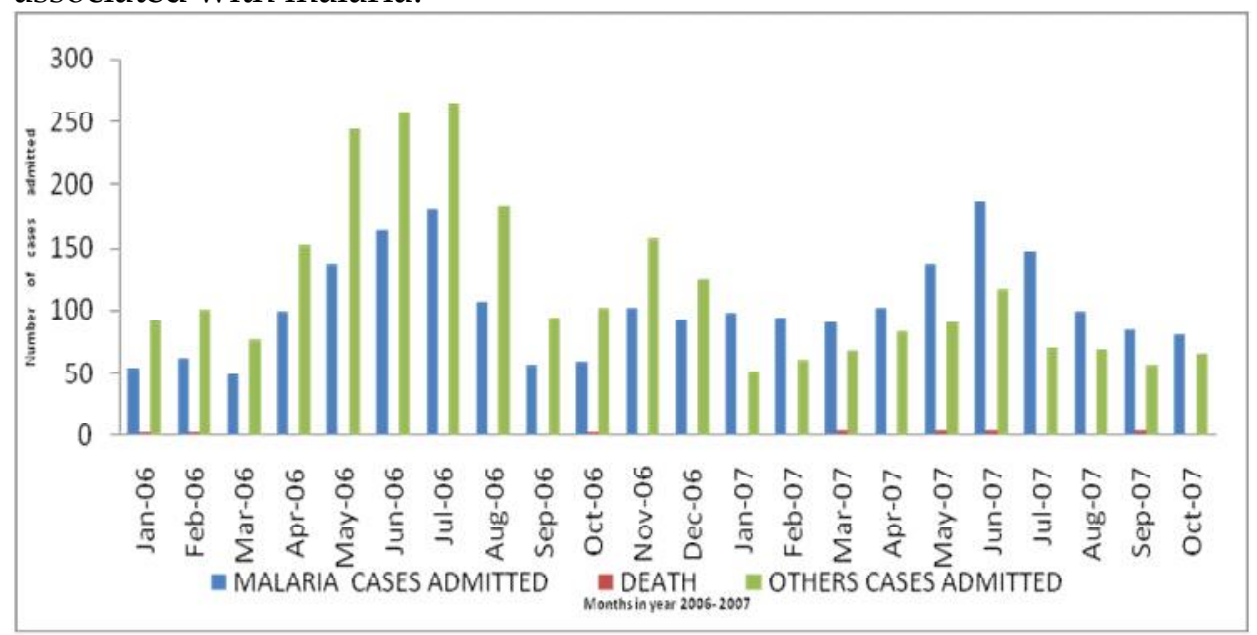

Figure 1: Cases admitted at Magugu HC in all age groups during 2006 - 2007 
Prospectively, during 2009 a total of 470 individuals (mean age $=17.9$ years) were included in the study (Table 1). Of the $<5$ years old children $42.9 \%(104 / 242)$ were reported to have fever $\left(\geq 37.5^{\circ} \mathrm{C}\right)$. Out of 180 children presenting with fever, $48.8 \%(88 / 180)$ were given antimalarial treatment, while $27.2 \%$ (49/190) were given antibiotics. Thirty two percent (42/130) of the individuals who received antimalarials had no fever, while the majority of those with fever $(67.7 \%)$ received antimalarials. Among individuals who presented with fever, $57.2 \%(103 / 180)$ were children under the age of five.

Table 1: Characteristics of the study population $(n=470)$

\begin{tabular}{ll}
\hline Variable & Measure \\
\hline Female $(\%(\mathrm{n} / \mathrm{N})$ & $56.4 \%(265 / 470)$ \\
Age in years, mean $\pm \mathrm{SD}$ & $17.9 \pm 22.28$ \\
$<5$ years & $51.4 \%(241 / 469)$ \\
$5-15$ years & $9.2 \%(43 / 469)$ \\
$>15$ years & $39.4 \%(185 / 469)$ \\
Bed net use & $90.5 \%(418 / 461)$ \\
Fever reported $\left(\geq 37.5^{\circ} \mathrm{C}\right) \%(\mathrm{n} / \mathrm{N})$ & $38.2 \%(180 / 470)$ \\
Residence in Magugu & $75.9 \%(356 / 469)$ \\
\hline
\end{tabular}

$\mathrm{SD}=$ Standard Deviation

Majority $(82.3 \%)$ of the patients who came to the Magugu HC had a history of having taken at least a medicament at home. Use of antimalarials prior to reporting at the health facility was reported by 160 of 470 (34\%) of individuals. A total of 122 individuals (25.9\%) took antibiotics, while 105 (22.3\%) took other treatments. Only 10.6\% (50/470) reported having not taken any medicine. Thirty three participants $(7 \%)$ could not remember having taken any medication prior visit to health facility.

Table 2: Blood smear results in relation to presence of fever, positive RDT and malaria clinical diagnosis among study participants

\begin{tabular}{llll}
\hline Variable & \multicolumn{2}{c}{ Blood slide } & P-Value \\
\cline { 2 - 3 } & Positive & Negative & \\
\hline Fever & 27 & 153 & $<0.001$ \\
RDT positive & 5 & 14 & 0.072 \\
Malaria clinical diagnosis & 29 & 307 & 0.156 \\
\hline
\end{tabular}

Out of 469 individuals who visited the health facilities, 373 (79.5\%) were clinically diagnosed as malaria. Of those who had history of fever, $88.8 \%(160 / 180)$ were clinically diagnosed as malaria cases. Of the 289 who had no fever, 213 patients (73.7 \%) were also clinically diagnosed as malaria cases. Medication history of the patients had an influence on the clinical diagnosis, since of those who reported taking antimalarial drugs prior to visiting the health facility, $40.2 \%(150 / 373)$ were clinically diagnosed as malaria cases. Malaria diagnosis based on microscopy was the gold 
standard, and 7.8\% (32/406) had a blood smear positive result. Only $16.1 \%(29 / 180)$ of the individuals presenting with fever had blood smear positive for malaria parasites. RDT was requested among 409 individuals, and 4.6\% (19/409) were positive. Twenty six per cent (5/19) of the RDT positive were also blood smear positive, while $6.6 \%(26 / 390)$ of the RDT negative were blood smear positive (Table 2). Using blood smear results as a gold standard, sensitivity of RDT was $16.1 \%$ and specificity of $96.2 \%$. The positive predictive value (PPV) was $26.3 \%$ and negative predictive value (NPV) $93.3 \%$.

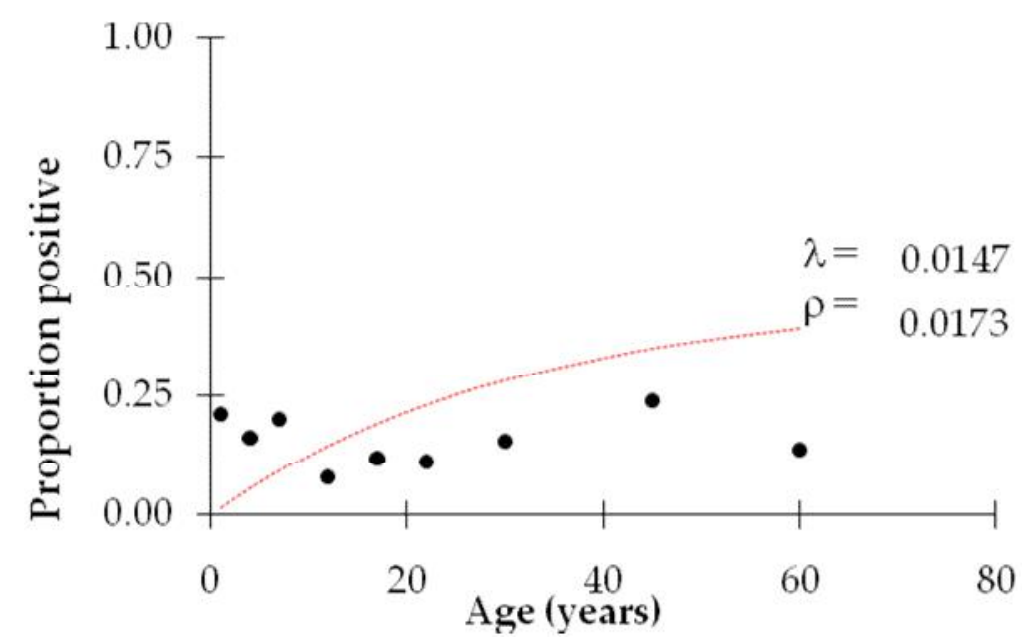

Figure 2: Seroprevalence of MSP-1 19 fitted into the model

A visit to the health facility ensured medication to be given to the individual since $94.3 \%(443 / 470)$ received any medicament. Some $42.8 \%$ of those who treated themselves with other treatments prior to the consultation were given antimalarial treatment. However, $20.6 \%(33 / 160)$ of individuals received again antimalarials. Of 130 individuals who received antimalarial treatment, $98(75.3 \%)$ had negative blood smears. This treatment was prescribed based on laboratory results. Bed-net use among participants was high at 90.5\% (418/462; Table 1). The majority 92.9\%(223/ 240) of children below five years slept under a bed net, and all those who were blood smear positive for malaria owned a bed net. The overall seroprevalence for MSP-1 19 was $16.9 \%$ and that for AMA-1 was 29.8\% (Figure 2,3). The seroprevalence showed a mild trend of increase with age in both antigens (NS). The pooled age group into three categories showed an increasing trend of seroprevalence and a fall in the older age groups. The seroprevalence for MSP- 119 by age category were as follows: <5years 18.5\% (42/227), 5 to 15 years $15.4 \%(8 / 39)$ and > 15 years $15.2 \%(26 / 171)$. For AMA-1 no clear gradient increase with age was observed: $<5$ years 31.7\% (72/227), 5 to 15 years $28.9 \%(11 / 38)$ and $>15$ years $27.5 \%$ (47/171). The EIR calculation using seroprevalence by AMA-1 (EIR 1.19) was higher than that of MSP-1 19 (EIR 0.20). The lambda $(\lambda)$ expressed MSP-1 19 was 0.015 while that expressed by AMA-1 was 0.033 . 


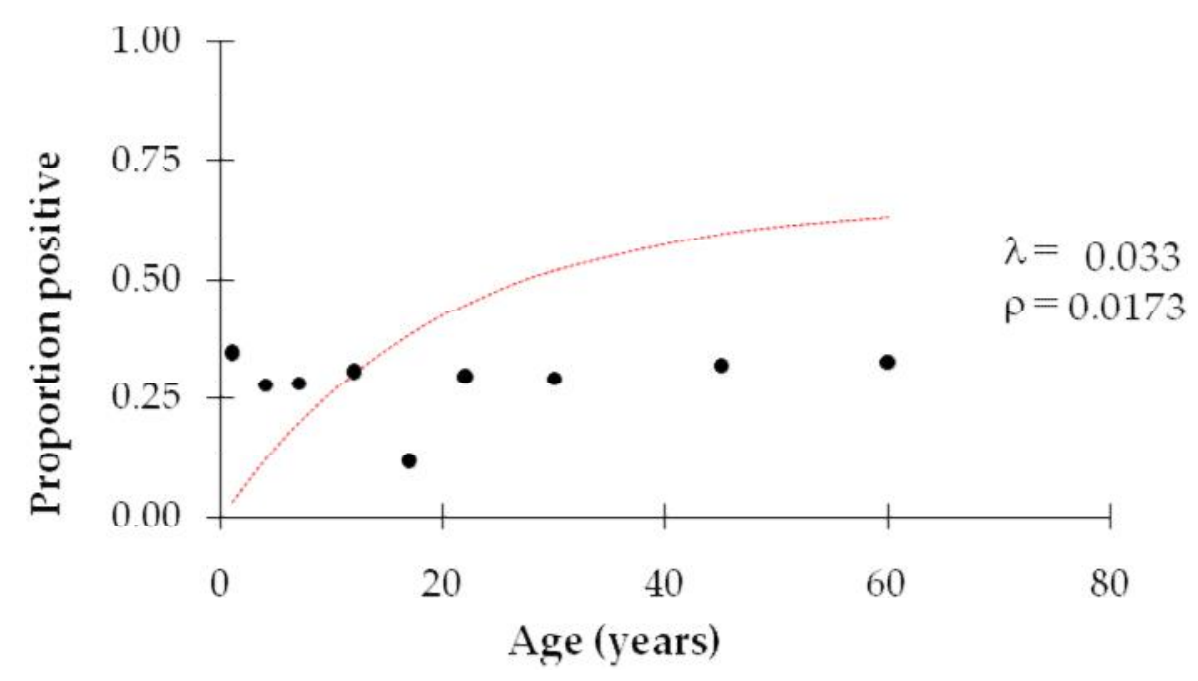

Figure 3: Seroprevalence of AMA-1 fitted into the seroprevalence model

\section{Discussion}

We found that only $7.6 \%$ of the malaria cases were confirmed slide positive, while $5.6 \%$ of the treated presumptive malaria cases were true malaria cases. This implies a significant overuse of ACT in the community as previously reported in another study in Tanzania (Nicastri et al., 2009). This irrational use of antimalarials puts further pressure on constrained resources and may promote development of malaria drug resistance. The majority of the patients who attended the health facility had a history of having taken a drug, mostly an antimalarial drug.

Attendance to the health facility could signify a poor response to treatment given at home. Awareness of malaria by the people in this area is very high, as reflected by practicing protective measures against malaria, including the use of bed nets and self medication against malaria in case of fever. Although the quality of the bed nets was not clear (whether treated or untreated), yet the majority claimed to be sleeping under a mosquito net. Similar results of recognizing fever and linking it with malaria among the caregivers/parents has been reported from elsewhere in Tanzania, Malawi and Kenya (Kaatano et al., 2006; Chibwana et al., 2009; Ye et al., 2009). The consequences of interpreting all fevers as being malaria are that other causes of febrile illness in the community are not recognised, resulting in delay of treatment (Rowe et al., 2006). Also it provides wrong statistics for malaria in terms of malaria morbidity and mortality.

Treatment of patients with antimalarials at the health facility depended on the following; clinical presentation, result of blood smear, history of fever and age of the patient. The majority of those smears positive were treated with antimalarials. Similarly, half of those with history of fever were treated with antimalarials. The majority of those $<5$ years were given antimalarials regardless of laboratory results. Fever in our study had $83 \%$ diagnostic sensitivity and $68 \%$ specificity. These findings were similar to findings by Msellem et al. (2009). Despite a negative blood smear result, three-quarters of the patients received antimalarial treatment. Similar results have been reported in elsewhere (Joshi et al., 2008; Fehintola \& Balogun, 2010). This practice calls for investigating other causes of fever (D'Acremont et al., 2010). 
While RDT is expected to decrease workload, increase cost-effectiveness and ensure accurate diagnosis of malaria, this test may not be applicable in an area with very low parasites circulating in the community as in this study area. Our findings indicate only 5 of the 32 individuals who were positive by microscopy, were also RDT positive. RDT sensitivity becomes low when parasite densities are below the level of 200 parasites/ $\mu \mathrm{L}$ or less than $0.002 \%$ of the red blood cells infected (WHO, 2010). Low parasite density of malaria observed in this study is likely to have contributed to the low sensitivity of the rapid test (Ochola et al., 2006). A few individuals were who were RDT positive were blood smear negative. This discordance between RDT and microscopy is due to the tendency of the RDT to remain positive weeks after parasite clearance (Hawkes \& Kain, 2007) due to persistence of malaria antigen in the circulation. This is likely to have resulted in the low sensitivity and high specificity observed in this study.

Serological markers can be used to determine the malaria transmission indices as observed in this study. The development of antibodies (and of acquired immunity) depends on the degree of exposure to infected mosquitoes. Application of the validated model relating age-specific seroconversion rates to EIR (Corran et al., 2007; Stewart et al., 2009), in our study area resulted into classifying Magugu area as a low transmission intensity area. This estimate correlates with the results of an entomological survey carried out in the nearby areas (Mwanziva et al., 2011). The low EIR in the area reflects the low number of immune individuals in the community as well as the low number of asymptomatic individuals (Shekalaghe et al., 2007), who are reservoirs of infection. Overall and age-wise, there was a difference in the observed seroprevalence of antibodies to MSP119 and AMA-1 antigens, the latter having the highest seroprevalence. Similar seroprevalence differences between the two antigens have been reported in other studies (Drakeley et al., 2005). At the age of 2-10 years there was increase in seroprevalence (results not shown) in both antigens used. Similar observations have been reported in Kenya (Williams et al., 2005) indicating that adaptive immunity in children is acquired between 2 and 10 years of age. Contrary to the findings of Steward et al. (2009) we did not observe a clear correlation between seroprevalence and age in this study. This is likely to be explained by the low transmission in the study area. As reported by Osier et al. (2008) a higher antibody response is observed among individuals with concurrent microscopic parasitaemia.

Also, bed net usage by the community, hence lack of constant exposure, plays a role (Targett, 1999). In addition, the small sample size may have lead to uneven distribution of individuals in different age categories. Whether migration of people into the study area who have not been recently or prior exposed to malaria, may have played a role in our findings could not be confirmed. It is likely that the serological results were not due to recent infection (Drakeley et al., 2005; Bousema et al., 2010). The low seroprevalence observed in the older age group could be explained by the slow sero-reversion rate of individuals of this age group (Drakeley et al., 2005). A similar observation has been reported by Stewart et al. (2009) in a low endemic area of northern Tanzania.

Since bed nets decrease human-vector contact, it has an effect on the infectivity rate of the mosquitoes and hence its chance to infect humans (Drame et al., 2010). This results into slow development of protective immunity, and eventually into severe malaria. Several factors were associated with this phenomenon including changes in physical environment (like climatic 
variation and land use) resulting into population displacement leading to importation of malaria in non-immune people (Suh, 2004).

In conclusion, Magugu area of northern Tanzania is of low malaria endemicity. However, there is substantial over-treatment, presumptive treatment, and self-treatment among the communities. Training of the medical personnel (Ssekabira et al., 2008) and community sensitization are necessary measures for the population to understand the burden of malaria in the area. Treatment of malaria should be based on microscopic confirmation, and where this is not possible, strictly designed clinical algorithms are needed so that treatment of malaria will become specific and precise. Use of RDT in areas where malaria endemicity is low, is of limited use.

\section{Acknowledgements}

We acknowledge the support of the Kilimanjaro Clinical Research Institute and Amani Medical Research Centre for providing technical assistance in sample processing and data analysis. Abdalla Jambia, Paul Mrema, Alutu Masokoto, Charles Lukwaro, Yokobeth are acknowledged for their support especially in field surveys and laboratory work. We are grateful to the village leaders for organizing participants to make field data collection possible and the study participants for their commitment and time. This study received financial support, from APRIORI Programme, Kilimanjaro Clinical Research Institute, KCM College and African Malaria Network.

\section{References}

Andersen, E., Jones, T. R., Purnomo, Masbar, S., Wiady, I., Tirtolusumo, S., Bangs, M. J., Charoenvit, Y., Gunawan, S., \& Hoffman, S. L. (1997) Assessment of age-dependent immunity to malaria in transmigrants. American Journal of Tropical Medicine and Hygiene 6, 647-649.

Barnes, K.I., Chanda, P. \& Ab, B.G. (2009) Impact of the large-scale deployment of artemether/lumefantrine on the malaria disease burden in Africa: case studies of South Africa, Zambia and Ethiopia. Malaria Journal 8 (Suppl 1) S8.

Besnard, P., Foumane, V., Foucher, J.F., Beliaud, P., Costa, J., Monnot, N., Le, M.J. \& Carnevale,P. (2006) Impact of a new parasitologic laboratory for malaria diagnosis on diagnosis and cost of malaria in a company setting: experience from Angola. Medicine Tropicale (Mars.) 66, 269-272.

Bhattarai, A., Ali, A.S., Kachur, S.P., Martensson, A., Abbas, A.K., Khatib, R., Al-Mafazy, A.W., Ramsan, M., Rotllant, G., Gerstenmaier, J.F., Molteni, F., Abdulla, S., Montgomery, S.M., Kaneko, A., \& Bjorkman, A. (2007) Impact of artemisinin-based combination therapy and insecticide-treated nets on malaria burden in Zanzibar. PLoS Medicine 4, e309.

Bousema, T., Youssef, R.M., Cook, J., Cox, J., Alegana, V. A., Amran, J., Noor, A.M., Snow, R. W.\& Drakeley, C. (2010) Serologic markers for detecting malaria in areas of low endemicity, Somalia, 2008. Emerging Infectious Diseases 16, 392-399.

Bruce-Chwatt, L.J. ( 1952) Malaria in African infants and children in Southern Nigeria. Annals of Tropical Medicine and Parasitology 2, 173-200. 
Chibwana, A.I., Mathanga, D.P., Chinkhumba, J. \& Campbell, C.H., Jr. (2009) Socio-cultural predictors of health-seeking behaviour for febrile under-five children in Mwanza-Neno district, Malawi. Malaria Journal 8, 219.

Corran, P., Coleman, P., Riley, E. \& Drakeley, C. (2007) Serology: a robust indicator of malaria transmission intensity? Trends in Parasitology 23, 575-582.

Corran, P.H., Cook, J., Lynch, C., Leendertse, H., Manjurano, A., Griffin, J., Cox, J., Abeku, T., Bousema, T., Ghani, A. C., Drakeley, C. \& Riley, E. (2008) Dried blood spots as a source of anti-malarial antibodies for epidemiological studies. Malaria Journal 7, 195.

Craig, M.H., Snow, R.W. \& Le, S.D. (1999) A climate-based distribution model of malaria transmission in sub-Saharan Africa. Parasitology Today 15, 105-111.

Crawley, J., Chu, C., Mtove, G., \& Nosten, F. (2010) Malaria in children. Lancet 375, 1468-1481. D'Acremont, V., Lengeler, C. \& Genton, B. (2010) Reduction in the proportion of fevers associated with Plasmodium falciparum parasitaemia in Africa: a systematic review. Malaria Journal 9, 240.

Drakeley, C.J., Corran, P.H., Coleman, P.G., Tongren, J.E., McDonald, S.L., Carneiro, I., Malima, R., Lusingu, J., Manjurano, A., Nkya, W.M., Lemnge, M.M., Cox, J., Reyburn, H. \& Riley, E. M. (2005) Estimating medium- and long-term trends in malaria transmission by using serological markers of malaria exposure. Proceedings of the National Academy of Sciences of the United States of America 102, 5108-5113.

Drame, P.M., Poinsignon, A., Besnard, P., Le, M.J., Dos-Santos, M.A., Sow, C.S., Cornelie, S., Foumane, V., Toto, J.C., Sembene, M., Boulanger, D., Simondon, F., Fortes, F., Carnevale, P. \& Remoue, F. (2010) Human antibody response to Anopheles gambiae saliva: an immunoepidemiological biomarker to evaluate the efficacy of insecticide-treated nets in malaria vector control. American Journal of Tropical Medicine and Hygiene 83, 115-121.

Elgayoum, S.M., El-Feki, A., Mahgoub, B.A., El-Rayah, E. \& Giha, H.A. (2009) Malaria overdiagnosis and burden of malaria misdiagnosis in the suburbs of central Sudan: special emphasis on artemisinin-based combination therapy era. Diagnostic Microbiology and Infectious Disease 64, 20-26.

Fehintola, F.A. \& Balogun, S.T. (2010) Malaria: passive case detection and healthcare providers' choices of chemotherapy. African Journal of Medicine and Medical Sciences 39, 49-54.

Franks, S., Baton, L., Tetteh, K., Tongren, E., Dewin, D., Akanmori, B. D., Koram, K.A., RanfordCartwright, L. \& Riley, E.M. (2003) Genetic diversity and antigenic polymorphism in Plasmodium falciparum: extensive serological cross-reactivity between allelic variants of merozoite surface protein. Infection and Immunity 71, 3485-3495.

Gosling, R.D., Drakeley, C.J., Mwita, A. \& Chandramohan, D. (2008) Presumptive treatment of fever cases as malaria: help or hindrance for malaria control? Malaria Journal 7, 132.

Greenwood, B.M., Bojang, K., Whitty, C.J. \& Targett, G.A. (2005) Malaria Lancet 365, 1487-1498.

Hawkes, M. \& Kain, K.C. (2007) Advances in malaria diagnosis Expert Review of Anti-infective Therapy 5, 485-495.

Hodel, E.M., Kabanywanyi, A.M., Malila, A., Zanolari, B., Mercier, T., Beck, H.P., Buclin, T., Olliaro, P., Decosterd, L.A. \& Genton, B. (2009) Residual antimalarials in malaria patients from Tanzania--implications on drug efficacy assessment and spread of parasite resistance. PLoS One 4, e8184. 
Joshi, R., Colford, J.M., Reingold, A.L. \& Kalantri, S. (2008) Non-malarial acute undifferentiated fever in a rural hospital in central India: diagnostic uncertainty and overtreatment with antimalarial agents. American Journal of Tropical Medicine and Hygiene 78, 393-399.

Kaatano, G.M., Muro, A.I. \& Medard, M. (2006) Caretaker's perceptions, attitudes and practices regarding childhood febrile illness and diarrhoeal diseases among riparian communities of Lake Victoria, Tanzania. Tanzania Health Research Bulletin 8, 155-161.

Luxemburger, C., Ricci, F., Nosten, F., Raimond, D., Bathet, S. \& White, N.J. (1997) The epidemiology of severe malaria in an area of low transmission in Thailand. Transactions of the Royal Society of Tropical Medicine and Hygiene 3, 256-262.

Maude, R.J., Pontavornpinyo, W., Saralamba, S., Aguas, R., Yeung, S., Dondorp, A.M., Day, N.P., White, N.J. \& White, L.J. (2009) The last man standing is the most resistant: eliminating artemisinin-resistant malaria in Cambodia. Malaria Journal 8, 31.

Msellem, M.I., Martensson, A., Rotllant, G., Bhattarai, A., Stromberg, J., Kahigwa, E., Garcia, M.,

Petzold, M., Olumese, P., Ali, A. \& Bjorkman, A. (2009) Influence of rapid malaria diagnostic tests on treatment and health outcome in fever patients, Zanzibar: a crossover validation study. PLoS Medicine 6, e1000070.

Murhandarwati, E.E., Black, C.G., Wang, L., Weisman, S., Koning-Ward, T.F., Baird, J.K., Tjitra, E., Richie, T.L., Crabb, B.S. \& Coppel, R.L. (2008) Acquisition of invasion-inhibitory antibodies specific for the 19-kDa fragment of merozoite surface protein 1 in a transmigrant population requires multiple infections. Journal of Infectious Diseases 8, 12121218.

Mwanziva, C., Shekalaghe, S., Ndaro, A., Mengerink, B., Megiroo, S., Mosha, F., Sauerwein, R., Drakeley, C., Gosling, R. \& Bousema, T. (2008) Overuse of artemisinin-combination therapy in Mto wa Mbu (river of mosquitoes), an area misinterpreted as high endemic for malaria. Malaria Journal 7, 232.

Mwanziva, C., Kitau, J., Tungu, P., Mweya, C., Mkali, H., Ndege, C., Sanga, A., Mtabho, C., Lukwaro, C., Azizi, S., Myamba, J., Chilongola, J., Magesa, S., Shekalaghe S. and Mosha, F. (2011) Transmission intensity and malaria vector population structure in Magugu, Babati District in northern Tanzania. Tanzania Journal of Health Research 13, 54-61.

Nicastri, E., Bevilacqua, N., Sane, S. M., Paglia, M.G., Meschi, S., Ame, S.M., Mohamed, J.A., Mangi, S., Fumakule, R., Di, C.A., Capobianchi, M.R., Kitua, A., Molteni, F., Racalbuto, V. \& Ippolito, G. (2009) Accuracy of malaria diagnosis by microscopy, rapid diagnostic test, and PCR methods and evidence of antimalarial overprescription in non-severe febrile patients in two Tanzanian hospitals. American Journal of Tropical Medicine and Hygiene 80, 712-717.

Nsimba, S.E. \& Rimoy, G.H. (2005) Self-medication with chloroquine in a rural district of Tanzania: a therapeutic challenge for any future malaria treatment policy change in the country. Journal of Clinical Pharmacology and Therapeutics 30, 515-519.

Ochola, L.B., Vounatsou, P., Smith, T., Mabaso, M.L. \& Newton, C.R. (2006) The reliability of diagnostic techniques in the diagnosis and management of malaria in the absence of a gold standard. Lancet Infectious Diseases 6, 582-588.

Osier, F.H., Fegan, G., Polley, S.D., Murungi, L., Verra, F., Tetteh, K.K., Lowe, B., Mwangi, T., Bull, P.C., Thomas, A.W., Cavanagh, D.R., McBride, J.S., Lanar, D.E., Mackinnon, M., Conway, 
D.J. \& Marsh, K. (2008) Breadth and magnitude of antibody responses to multiple Plasmodium falciparum merozoite antigens are associated with protection from clinical malaria. Infection and Immunity 76, 2240-2248.

Rowe, A.K., Rowe, S.Y., Snow, R.W., Korenromp, E.L., Schellenberg, J.R., Stein, C., Nahlen, B.L.,Bryce, J., Black, R.E. \& Steketee, R. W. (2006) The burden of malaria mortality among African children in the year 2000. International Journal of Epidemiology 35, 691-704.

Shekalaghe, S.A., Bousema, J.T., Kunei, K.K., Lushino, P., Masokoto, A., Wolters, L.R., Mwakalinga, S., Mosha, F.W., Sauerwein, R.W. \& Drakeley, C.J. (2007) Submicroscopic Plasmodium falciparum gametocyte carriage is common in an area of low and seasonal transmission in Tanzania. Tropical Medicine \& International Health 12, 547-553.

Ssekabira, U., Bukirwa, H., Hopkins, H., Namagembe, A., Weaver, M.R., Sebuyira, L.M., Quick, L., Staedke, S., Yeka, A., Kiggundu, M., Schneider, G., McAdam, K., Wabwire-Mangen, F. \& Dorsey, G. (2008) Improved malaria case management after integrated team-based training of health care workers in Uganda. American Journal of Tropical Medicine and Hygiene 79, 826-833.

Stewart, L., Gosling, R., Griffin, J., Gesase, S., Campo, J., Hashim, R., Masika, P., Mosha, J., Bousema, T., Shekalaghe, S., Cook, J., Corran, P., Ghani, A., Riley, E.M. \& Drakeley, C. (2009) Rapid assessment of malaria transmission using age-specific sero-conversion rates. PLoS One 4, e6083.

Suh, K.N., Kain, K.C. \& Keystone, J.S. (2004) Malaria. Canadian Medical Association Journal 170, 1693-1702.

Targett, G. (1999) Vaccine efficacy, and immunity affecting transmission. Parassitologia 41, 433-436. Warsame, M., Wernsdorfer, W. H., Perlmann, H., Lebbad, M., Ericsson, O., Matola, Y. G., TroyeBlomberg, M., Perlmann, P. \& Berzins, K. (1997) A malariometric survey in a rural community in the Muheza district, Tanzania: age profiles in the development of humoral immune responses. Acta Tropica 2, 239-253.

White, N. J. (2010) Artemisinin resistance--the clock is ticking. Lancet, 9758, 2051-2052.

Williams, T.N., Mwangi, T.W., Wambua, S., Alexander, N.D., Kortok, M., Snow, R.W. \& Marsh, K. (2005) Sickle cell trait and the risk of Plasmodium falciparum malaria and other childhood diseases. Journal of Infectious Diseases 192, 178-186.

WHO (2010) Malaria Rapid Diagnostic Test Performance: Executive Summary, Results of WHO Product Testing Of Malaria RDTs: Round 2 (2009). World Health Organization, Geneva.

WHO (2009) World Malaria Report 2009. World Health Organization, Geneva.

Ye, Y., Madise, N., Ndugwa, R., Ochola, S. \& Snow, R. W. (2009) Fever treatment in the absence of malaria transmission in an urban informal settlement in Nairobi, Kenya. Malaria Journal 8, 160. 